\title{
Identificación y perfil de sensibilidad de Candida spp. aisladas de hemocultivos en hospitales de Paraguay
}

\author{
Gustavo Aguilar ${ }^{1}$, Patricia Araujo², Graciela Lird ${ }^{3}$, Sonia Insaurralde ${ }^{4}$, Aníbal Kawabata ${ }^{5}$, Edelira Ayala ${ }^{6}$, \\ Juan Irala7 y Rocío Argüello ${ }^{8}$
}

Forma de citar

Aguilar G, Araujo P, Lird G, Insaurralde S, Kawabata A, Ayala E, et al. Identificación y perfil de sensibilidad de Candida spp. aisladas de hemocultivos en hospitales de Paraguay. Rev Panam Salud Publica. 2020;44:e34. https://doi.org/10.26633/ RPSP.2020.34

RESUMEN

Objetivo. Reportar las especies identificadas en candidemias aisladas en hospitales del país, su distribución según los grupos etarios y determinar la sensibilidad a fluconazol, voriconazol y anfotericina B.

Métodos. Estudio retrospectivo de candidemias reportados por siete hospitales al Laboratorio Central de Salud Pública en el período 2010 - 2018. La identificación y el antifungigrama fueron realizados en el sistema automatizado Vitek ${ }^{\circledR 2}$.

Resultados. Se informaron 520 casos de candidemias. Las especies prevalentes fueron Candida albicans $(34,4 \%)$, C. parapsilosis (30,4\%), C. tropicalis (25,4\%), C. glabrata (4,8\%), C. krusei (2,1\%). Otras menos frecuentes fueron: C. Iusitaniae (1,2\%), C. guilliermondii (1\%), C. famata (0,3\%), C. rugosa $(0,2 \%)$ y C. kefyr $(0,2 \%)$. Las candidemias en ancianos $(48,5 \%)$ y adultos $(29,9 \%)$ fueron las más prevalentes. $C$. parapsilosis fue más frecuente en recién nacidos y lactantes y $C$. tropicalis en pacientes pediátricos. El 2,8 \% y 0,6\% de C. albicans y el $4,4 \%$ y $2,5 \%$ de $C$. parapsilosis fueron resistentes a fluconazol y voriconazol, respectivamente. El $8 \%$ de $C$. glabrata fue resistente a fluconazol y dos aislamientos presentaron una concentración inhibitoria mínima para voriconazol mayor que $0,25 \mathrm{mg} / \mathrm{L}$

Conclusiones. Este es el primer reporte de candidemias en hospitales de Paraguay que, junto a otros estudios, permitirá un mejor conocimiento de esta infección en el país.

Palabras clave Candidemia; antifúngicos; fluconazol; voriconazol; Paraguay.

La candidemia es un problema global con un gran impacto en los pacientes. Según algunas publicaciones, la tasa de mortalidad en terapia intensiva puede llegar a más de 50\% (1-3). El conocimiento de la epidemiología en cuanto a especies, grupo etario y sensibilidad a los antifúngicos es fundamental para tomar medidas de control y tratamiento (4). En América Latina, varios países han reportado datos sobre candidemias (5-9). Candida albicans es la especie más prevalente, seguida por C. parapsilosis, C. tropicalis y C. glabrata. Estas constituyen más de $90 \%$ de los aislamientos con una resistencia baja a los

\footnotetext{
Instituto de Previsión Social, Hospital Central, Asunción, Paraguay. Gustavo Aguilar, $\triangle$ gaguifer@hotmail.com

2 Laboratorio Central de Salud Pública, Ministerio de Salud, Asunción, Paraguay.

3 Hospital de Clínicas, San Lorenzo, Paraguay.

4 Hospital Nacional de Itaguá, Itaguá, Paraguay.
}

antifúngicos de uso común. La distribución de las especies según los grupos etarios varía según el país por diversos factores (10).

En Paraguay, no existen datos publicados sobre este tipo de infección. Los objetivos de este trabajo son mostrar las especies de Candida aisladas en pacientes a partir de hemocultivos procedentes de los siete principales hospitales de Paraguay en los últimos nueve años y conocer la sensibilidad al fluconazol, voriconazol y anfotericina B de las especies y la distribución de estas según grupos etarios.

\footnotetext{
5 Hospital del Trauma "Prof. Dr. Manuel Giagni”, Asunción, Paraguay.

6 Instituto de Medicina Tropical, Asunción, Paraguay.

7 Hospital Militar, Asunción, Paraguay.

8 Hospital Pediátrico "Niños de Acosta Nu", San Lorenzo, Paraguay.
} 


\section{MATERIALES Y MÉTODOS}

Estudio retrospectivo de casos de fungemias por Candida spp. reportados al Laboratorio Central del Ministerio de Salud Pública de siete hospitales de Paraguay: Hospital Central del Instituto de Previsión Social, Hospital Nacional de Itaguá, Hospital de Clínicas, Instituto de Medicina Tropical, Hospital Pediátrico "Niños de Acosta Nu", Hospital Militar y el Hospital del Trauma "Prof. Dr. Manuel Giagni" en el período de enero 2010 a diciembre 2018.

Los aislamientos fueron remitidos con formularios en los que se especificó el nombre, edad y sexo del paciente, el género y especie de levadura y el antifungigrama (estos dos últimos datos según la disponibilidad del hospital). Para el estudio se tuvo en cuenta a pacientes diferentes de una sola muestra. La identificación y el antifungigrama de los aislamientos se realizaron en el Laboratorio Central de Salud Pública con el sistema automatizado Vitek ${ }^{\circledR 2}$ (bioMérieux, Francia). Para complementar la identificación se realizaron métodos fenotípicos como la morfología de las levaduras en CHROM agar Candida Medium ${ }^{\circledR}$ (Difco, Estados Unidos de América), caldo de extracto de malta (Oxoid, England), microcultivo en agar maíz Tween 80, prueba de filamentación y prueba de inhibición en agar Sabouraud $\mathrm{ClNa}$ 6,5\% (Britania, Argentina), auxonograma con hidratos de carbono (glucosa, sacarosa, maltosa, trehalosa, lactosa, celobiosa, arabinosa, rafinosa, galactosa) y pruebas de crecimiento en agar Sabouraud (Britania, Argentina) a 28, $37 \mathrm{y}$ $42^{\circ} \mathrm{C}$ por 48 horas. Los aislamientos fueron almacenados a temperatura ambiente en agua estéril por duplicado en el cepario de la sección de Micología del Laboratorio Central de Salud Pública.

Para la identificación en el sistema Vitek ${ }^{\circledR} 2$ se utilizaron las tarjetas YST (bioMérieux, Francia), que tienen pruebas bioquímicas que incluyen asimilación de hidratos de carbono y ácidos orgánicos. Para el antifungigrama, se utilizaron las tarjetas AST-YS07 (bioMérieux, Francia), que contienen un panel de diluciones seriadas de fluconazol (1 a $64 \mathrm{mg} / \mathrm{L}$ ), voriconazol $(0,12$ a $8 \mathrm{mg} / \mathrm{L})$ y anfotericina B $(0,25$ a $16 \mathrm{mg} / \mathrm{L})$. Se utilizaron C. parapsilosis ATCC 22019 y C. krusei ATCC 6258 como controles de calidad para las tarjetas AST YS07.

Para definir la sensibilidad según la concentración inhibitoria mínima (CIM), se utilizaron los puntos de corte propuestos en el documento M60 (11) del Instituto de Normas Clínicas y de Laboratorio de los Estados Unidos (CLSI, por sus siglas en inglés) para las especies C. albicans, C. parapsilosis, C. tropicalis, C. glabrata y C. krusei. Se consideró resistente al fluconazol una CIM $\geq 8 \mathrm{mg} / \mathrm{L}$ para las tres primeras anteriores y una $\mathrm{CIM} \geq 64 \mathrm{mg} / \mathrm{L}$ para $C$. glabrata. Para el voriconazol, se consideró resistente una CIM $\geq 2 \mathrm{mg} / \mathrm{L}$ para C. krusei y una CIM $\geq 1 \mathrm{mg} / \mathrm{L}$ para C. albicans, C. parapsilosis y C. tropicalis.

Para los puntos de corte epidemiológicos (EVC, por sus siglas en inglés), se utilizó el documento M59 (12) del CLSI donde categoriza los aislamientos en tipo silvestre (WT, por sus siglas en inglés) ( $\leq 2 \mathrm{mg} / \mathrm{L}$ ) o no tipo silvestre (no WT) $(>2 \mathrm{mg} / \mathrm{L})$ para la anfotericina B y la CIM $\leq 0,25 \mathrm{mg} / \mathrm{L}$ para el tipo silvestre (WT) o no tipo silvestre (no WT) $(>0,25$ $\mathrm{mg} / \mathrm{L}$ ) para el voriconazol. El WT indica que el aislamiento no posee ninguna mutación que afecte la sensibilidad al antifúngico.
Los grupos etarios se definieron: recién nacidos $(<28$ días de edad), lactantes (28 días a 2 años), niños (> 2 a $\leq 18$ años), adultos ( $>18$ a 60 años) y ancianos ( $>60$ años).

Para los cuadros y el análisis de los datos se utilizó el programa Excel $^{\circledR} 2010$ (Microsoft).

Este trabajo fue aprobado por el comité de ética del Laboratorio Central de Salud Pública. Solo los investigadores tuvieron acceso a los datos de los pacientes, que permanecieron confidenciales y codificados durante el estudio.

\section{RESULTADOS}

Durante el período estudiado, se reportaron 520 fungemias por Candida spp. en siete de los principales hospitales de Paraguay. La prevalencia por trienios según las especies no cambió en el período de estudio (cuadro 1). Candida albicans fue el principal agente con $34,4 \%(n=179)$ de los casos. Las especies no C. albicans constituyeron el 65,6\% $(n=341)$ con el siguiente orden de prevalencia: C. parapsilosis $30,4 \%(n=158)$, C. tropicalis $25,4 \%$ $(n=132)$, C. glabrata $4,8 \%(n=25)$ y otras especies $5 \%(n=26)$. Entre estas, encontramos C. krusei 2,1\% $(n=11)$, C. lusitaniae $1,2 \%(n=6)$, C. guilliermondii $1 \%(n=5)$, dos aislamientos de C. famata y uno de C. rugosa y C. kefyr, respectivamente.

El promedio de edad fue de 49 años (0 - 99 años). Los casos fueron más frecuentes en pacientes de sexo masculino (relación masculino: femenino $=286: 234)$. La distribución de las especies según el grupo etario se muestra en el cuadro 2. Del total de aislamientos, hubo predominio en ancianos y adultos con $48,5 \%$ $(n=252)$ y $29,9 \%(n=155)$, respectivamente. En ambos grupos etarios, el orden de prevalencia fue C. albicans, C. parapsilosis, C. tropicalis y C. glabrata. En los pacientes pediátricos, C. tropicalis fue la especie más aislada, 39,4\% $(n=13)$. En recién nacidos y lactantes prevaleció C. parapsilosis con $51,1 \%(n=24)$ y $45,5 \%$ $(n=15)$, respectivamente. Noventa y uno por ciento $(n=10)$ de las C. krusei y todas las C. glabrata $(\mathrm{n}=25)$ se aislaron en adultos $\mathrm{y}$ ancianos.

En el cuadro 3 se muestran la CIM de las Candida spp. testadas en el sistema automatizado Vitek ${ }^{\circledR}$. Todas las levaduras de este estudio consideradas en el documento M59 no sobrepasaron el valor de $2 \mathrm{mg}$ /L para la anfotericina B. Se las consideró, entonces, como tipos silvestres para este antifúngico.

Se encontró que 4,4\% $(n=7)$ de las C. parapsilosis, $2,8 \%(n=5)$ de las C. albicans y $8 \%(n=2)$ de las C. glabrata fueron resistentes a fluconazol y que $2,5 \%(n=4)$ de las $C$. parapsilosis y un aislamiento $(0,6 \%)$ de $C$. albicans fueron resistentes al voriconazol.

CUADRO 1. Distribución de 520 Candida spp. aisladas de hemocultivos según trienios (2010-2018)

\begin{tabular}{lcccccc} 
& & \multicolumn{5}{c}{ Especies $n$} \\
\cline { 3 - 7 } Trienio & $n$ & $\begin{array}{c}\text { C. } \\
\text { albicans }\end{array}$ & $\begin{array}{c}\text { C. } \\
\text { parapsilosis }\end{array}$ & $\begin{array}{c}\text { C. } \\
\text { tropicalis }\end{array}$ & $\begin{array}{c}\text { C. } \\
\text { glabrata }\end{array}$ & $\begin{array}{c}\text { Otras } \\
\text { Candida } \\
\text { spp. }{ }^{1}\end{array}$ \\
$2010-2012$ & 147 & 50 & 53 & 28 & 10 & 6 \\
$2013-2015$ & 145 & 50 & 41 & 42 & 2 & 10 \\
$2016-2018$ & 228 & 79 & 64 & 62 & 13 & 10 \\
Total $(\%)$ & & $179(34,4)$ & $158(30,4)$ & $132(25,4)$ & $25(4,8)$ & $26(5)$ \\
\hline
\end{tabular}

$n$ : número de especies aisladas.

${ }^{1}$ Otras Candida spp. incluye: $C$. krusei $(n=11)$, C. Iusitaniae $(n=6)$, C. guilliermodii $(n=5)$, C. famata $(n=2)$, C. rugosa $(n=1)$, C. $\operatorname{kefyr}(n=1)$.

Fuente: elaboración propia a partir de datos obtenidos en el estudio. 
CUADRO 2. Distribución de 520 Candida spp. aisladas de hemocultivos según grupos etarios

\begin{tabular}{|c|c|c|c|c|c|c|}
\hline \multicolumn{2}{|c|}{ Pacientes } & \multicolumn{5}{|c|}{ Especies $n(\%)$} \\
\hline Edad & $n$ & C. albicans & C. parapsilosis & C. tropicalis & C. glabrata & $\begin{array}{l}\text { Otras Candida } \\
\text { spp. }{ }^{1}\end{array}$ \\
\hline$\leq 28$ días & 47 & $15(8,3)$ & $24(15,2)$ & $7(5,3)$ & 0 & $1(3,8)$ \\
\hline$>2$ años $-\leq 18$ años & 33 & $10(5,6)$ & $7(4,4)$ & $13(9,8)$ & 0 & $3(11,5)$ \\
\hline$>18$ años $-\leq 60$ años & 155 & $60(33,5)$ & $41(25,9)$ & $34(25,8)$ & $8(32)$ & $12(46,2)$ \\
\hline
\end{tabular}

$n$ : número de especies aisladas.

${ }^{1}$ Otras Candida spp. incluye: C. krusei $(n=11)$, C. Iusitaniae $(n=6)$, C. guilliermodii $(n=5)$, C. famata $(n=2)$, . rugosa $(n=1)$, C. kefyr $(n=1)$

Fuente: elaboración propia a partir de datos obtenidos en el estudio.

CUADRO 3. Sensibilidad in vitro de 520 Candida spp. determinada mediante el sistema Vitek ${ }^{\circledR 2}$

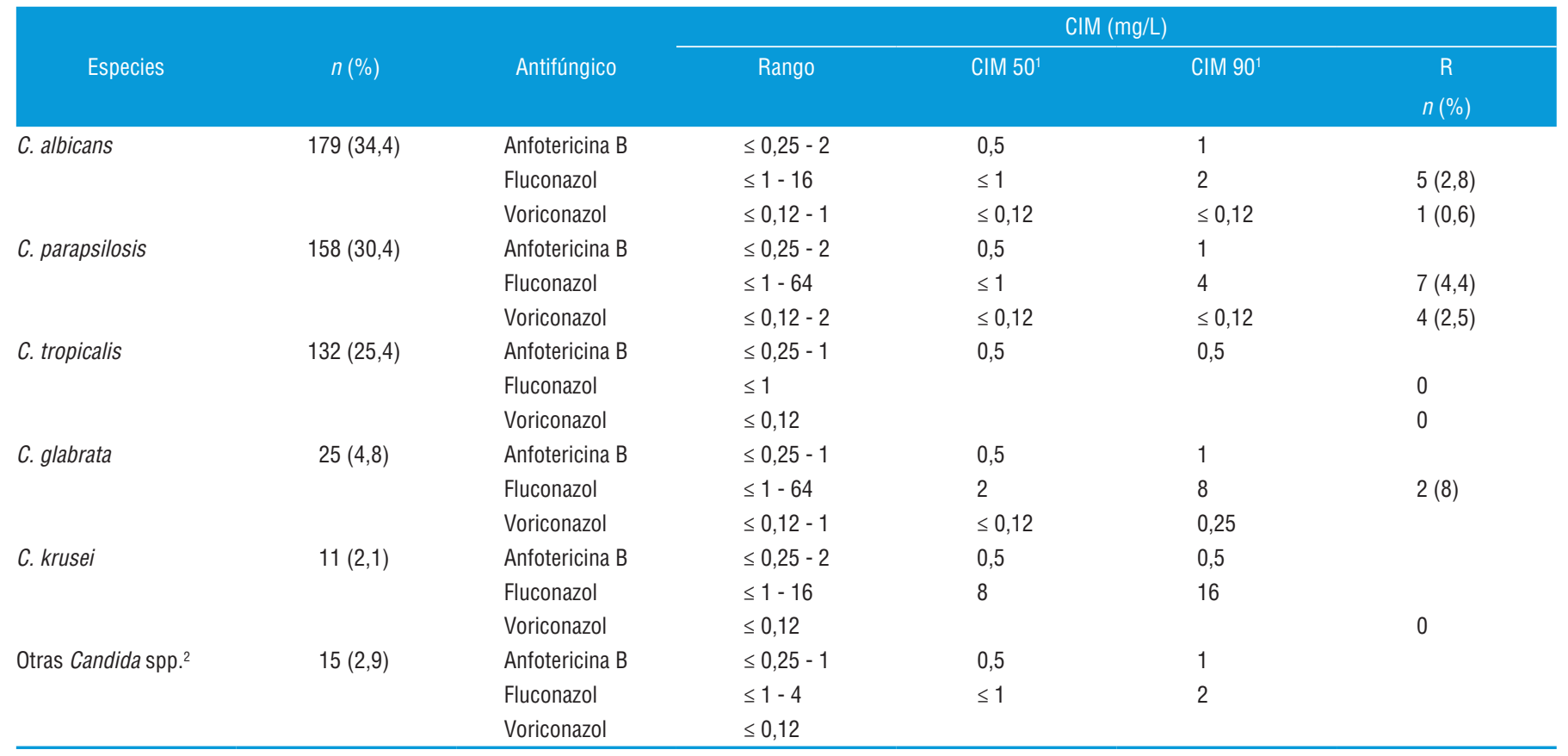

n: número de aislamientos; CIM: Concentración inhibitoria mínima; R: resistente.

n: numero de aislamientos; CIM: Concentración inhibitoria minima; R: resister
${ }^{1} \mathrm{CIM} 50$ y 90: CIM en el que $50 \%$ y $90 \%$ de los aislamientos son inhibidos.

${ }^{2}$ Otras Candida spp. incluye: C. lusitaniae $(n=6)$, C. guilliermodii $(n=5)$, C. famata $(n=2)$, C. rugosa $(n=1)$, C. $k e f y r(n=1)$

Fuente: elaboración propia a partir de datos obtenidos en el estudio.

Además,se identificaron dos aislamientos (8\%) de C. glabrata no tipo silvestre para voriconazol (CIM $>0,25 \mathrm{mg} / \mathrm{L})$.

\section{DISCUSIÓN}

El programa de vigilancia de resistencia a los antifúngicos SENTRY (1997 - 2016) informa que las candidemias para las cuatro especies más prevalentes en América Latina son causadas por C. albicans seguida por C. parapsilosis, C. tropicalis y C. glabrata (13). En nuestros datos se observó esta misma relación, que se mantuvo en el período de estudio (cuadro 1). Lo mismo ocurre en Argentina, Brasil, Colombia, Chile y Perú (5, 7-9, 14, 15). En cambio, en Costa Rica, México y Venezuela, C. parapsilosis es la más prevalente seguida por C. albicans $(6,16,17)$.

En C. albicans, el porcentaje de resistencia a fluconazol de $2,8 \%$ que observamos fue similar en Perú, aunque menor en Chile y demás países de América Latina $(5,6,9,14,15)$.
C. parapsilosis fue el segundo aislamiento más frecuente y el primero entre los recién nacidos y lactantes de este estudio. Esta especie es la que presentó mayor resistencia a fluconazol y voriconazol (4,4\% y 2,5\%, respectivamente). Porcentajes similares se informan en Perú e inferiores en Argentina y Chile (5, 14, 15). La presencia de esta especie se relaciona con el mal manejo del catéter en los pacientes y constituye así una señal de alerta para mejorar las prácticas de uso en estos dispositivos y evitar la transmisión vertical (18).

C. tropicalis fue la tercera especie más aislada y la más frecuente en pacientes pediátricos de nuestra serie. En Perú y Venezuela, es más frecuente en personas mayores de 60 años $(6,15)$. El 100\% de los aislamientos de C. tropicalis que reportamos fueron sensibles a fluconazol y voriconazol, muy similar a la epidemiología América Latina (6, 9, 13-15).

En nuestra serie, la resistencia a fluconazol en C. glabrata fue de $8 \%$, similar a la encontrada en Venezuela, aunque menor 
que Argentina y mayor que Chile $(5,6,14)$. Es de destacar que recuperamos C. glabrata solo de adultos y ancianos. Esto es similar a otros reportes donde hay un aumento proporcional del número de casos por C. glabrata con la edad $(9,19)$. Nuestros aislamientos de esta especie mostraron una CIM $90 \leq 8 \mathrm{mg} / \mathrm{L}$ para fluconazol; esto permite utilizar este fármaco para esta levadura en esquemas de tratamiento a dosis altas (20).

En este trabajo, aislamos C. krusei en 2,1\% de los casos. Esto representa el doble en comparación con los reportes de Argentina, Brasil, Colombia y Venezuela $(5-8,21)$. C. krusei y otras menos frecuentes como C. lusitaniae, C. guilliermondii, C. kefyr, C. famata y C. rugosa se aislaron en $77 \%$ de la población de adultos y ancianos de nuestra serie. En Venezuela, 90\% de estas levaduras fueron aisladas en menores de un año (6). El programa de vigilancia SENTRY relaciona estas especies con bajas sensibilidad a fluconazol, en especial la C. guilliermondii, reportada en mayor proporción en América Latina (22). En nuestro estudio, la CIM 90 para C. guilliermondii para fluconazol fue $2 \mathrm{mg} / \mathrm{L}$. Estas especies poco frecuentes no están contempladas en los protocolos de tratamiento y son emergentes, por ello es importante su seguimiento para conocer su epidemiología (20).

Las debilidades del trabajo serían la falta de datos de los pacientes en cuando a comorbilidades, unidad de cuidados intensivos o servicio del cual se deriva el caso, tiempo de internación y tratamiento, entre otros. Esto ayudaría a correlacionar mejor a los aislamientos y su sensibilidad a antifúngicos en la toma de decisiones en los centros. Además, algunos hospitales reportaron los casos con mayor regularidad;esto permite que sean más representados en los datos. Otro punto, relacionado con los casos de CIM alta, es que, a pesar de que fueron corroborados nuevamente en el Laboratorio Central de Salud Pública en el sistema Vitek ${ }^{\circledR} 2$, que tiene muy buena correlación con el método de referencia de microdilución $(23,24)$, es muy importante realizar este método de referencia a los aislamientos resistentes para verificar.

En conclusión, este es el primer trabajo publicado sobre candidemias en Paraguay que muestra una prevalencia de especies similares a los reportes en otros países de América Latina. Encontramos una resistencia global para el fluconazol y voriconazol del 3,6\%. Casi 50\% de los casos ocurrieron en mayores de 60 años. Es importante señalar que, debido al alerta epidemiológica por C. auris emitida para América Latina en el 2016 por la Organización Panamericana de la Salud, se conformó la Red de Micología del Ministerio de Salud en el país (25). Esto permitirá reforzar la vigilancia, incluir más instituciones y aumentar las capacitaciones en temas de infecciones por levaduras, para conocer mejor la epidemiología de estas micosis.

Contribuciones de los autores. GA participó en el diseño, análisis e interpretación de los datos y escritura del manuscrito. PA participó en el diseño, escritura del manuscrito, e interpretación y corrección de los datos. GL, SI, AK, EA, JI y RA se dedicaron al envío de los datos y revisión del manuscrito.Todos los autores leyeron y aprobaron las correcciones del manuscrito.

Conflicto de intereses. Ninguno declarado por los autores.

Declaración. Las opiniones expresadas en este manuscrito son responsabilidad del autor y no reflejan necesariamente los criterios ni la política de la RPSP/PAJPH y / o de la OPS.

\section{REFERENCIAS}

1. Colombo AL, Nucci M, Park BJ, Nouér SA, Arthington-Skaggs B, da Matta DA, et al. Epidemiology of candidemia in Brazil: a nationwide sentinel surveillance of candidemia in eleven medical centers. J Clin Microbiol. 2006;44(8):2816-23.

2. Bassetti M, Righi E, Ansaldi F, Merelli M, Trucchi C, De Pascale G, et al. A multicenter study of septic shock due to candidemia: outcomes and predictors of mortality. Intensive Care Med. 2014;40(6):839-45.

3. Lortholary O, Renaudat C, Sitbon K, Madec Y, Denoeud-Ndam L, Wolff $\mathrm{M}$, et al. Worrisome trends in incidence and mortality of candidemia in intensive care units (Paris area, 2002-2010). Intensive Care Med. 2014;40(9):1303-12.

4. Eliakim-Raz N, Babaoff R, Yahav D, Yanai S, Shaked H, Bishara J. Epidemiology, microbiology, clinical characteristics, and outcomes of candidemia in internal medicine wardsa retrospective study. Int J Infect Dis. 2016;52:49-54.

5. Córdoba S, Vivot W, Bosco-Borgeat ME, Taverna C, Szusz W, Murisengo $\mathrm{O}$, et al.Species distribution and susceptibility profile of yeast isolated from blood cultures: results of a multicenter active laboratory based surveillance study in Argentina. Rev Argent Microbiol. 2011; 43(3):176-85.

6. Panizo M, Reviákina V, Dolande M, Selgrad S. Candida spp. in vitro susceptibility profile to four antifungal agents. Resistance surveillance study in Venezuelan strains. Med Mycol. 2009; 47(2):137-43.

7. Castro L, Schütze M, Bücker D, Vasconcellos L. Prevalence of fungemia in a tertiary hospital: Analysis of the last decade. Rev Assoc Med Bras. 2016;62(4):315-19.

8. Cortés J, Jaimes J, Leal A. Incidencia y prevalencia de candidemia en pacientes críticamente enfermos en Colombia. Rev Chil Infectol. 2013;30(6):599-604.
9. Nucci M, Queiroz-Telles F, Alvarado-Matute T, Tiraboschi I, Cortes $\mathrm{J}$, et al. Epidemiology of candidemia in Latin America: alaboratory-based survey. PlosOne. 2013;8(3):e59373.

10. Hesstvedt L, Arendrup MC, Poikonen E, Klingpor L, Friman V, Nordøy I, et al. Differences in epidemiology of candidemia in the Nordic countries: what is to blame? Mycoses. 2017;60:11-19.

11. Clinical and Laboratory Standards Institute. Document M60. Performance standards for antifungal susceptibility testing of yeasts. $1^{\circ}$ ed.Wayne, PA: CLSI; 2017.

12. Clinical and Laboratory Standards Institute. Document M59. Epidemiological cutoff values for antifungal susceptibility testing. $1^{\circ}$ ed.Wayne, PA: CLSI; 2016.

13. Pfaller MA, Diekema DJ, Turnidge JD, Castanheira M, Jones RN Twenty years of the SENTRY Antifungal Surveillance Program: results for Candida species from 1997-2016. Open Forum Infect Dis. 2019;6(S1):S79-S94

14. Santolaya ME, Thompson L, Benadof D, et al. A prospective, multi-center study of Candida bloodstream infections in Chile. Plos One. 2019;14(3):e212924.

15. Rodriguez L, Bustamante B, Huaroto L, et al. A multi-centric study of Candida bloodstream infection in Lima-Callao, Perú: species distribution, antifungal resistance and clinical outcomes. Plos One. 2017;12(4):e0175172.

16. Villalobos J, Castro J, Avilés Á, Peláez M, Somogyi P, Sandoval L. Epidemiología de la candidemia en el Hospital México. Acta Med Costarric. 2016;58(1):15-21.

17. Villalobos J, Castro J, Avilés A, Peláez M, Somogyi T, Sandoval L. Candida parapsilosis: principal causa de candidemia en un hospital de referencia para adultos de Costa Rica. Rev Chil Infectol. 2016;33(2):159-65. 
18. Abid-Said D, Anaissie E, Uzun O, Raad I, Piazcowsky H,Vartivarian $\mathrm{S}$. The epidemiology of hematogenous candidiasis caused by different Candida species. Clin Infect Dis. 1997;24:1122-8.

19. Malani A, Hmoud J, Chiu L, Carver PL, Bielaczyc A, et al. Candida glabrata fungemia: experience in a tertiary care center. Clin Infect Dis. 2005;41:975-81.

20. Pappas PG, Kauffman CA, Andes DR, et al. Clinical practice guideline for the management of candidiasis: 2016 update by the Infectious Diseases Society of America. Clin Infect Dis. 2016;62(4):e1-e50.

21. Bonfietti L, Szeszs M, Chang M, Martins M, Pukinskas S, Nunes M, Pereira G, Paniago A et al. Ten year study of species distribution and antifungal susceptibilities of Candida bloodstream isolates at a Brazilian tertiary hospital. Mycopathologia. 2012; 174(5-6): 389-96.

22. Pfaller MA, Castanheira M, Messer SA, Moet GJ, Jones RN. Variation in Candida spp. distribution and antifungal resistance rates among bloodstream infection isolates by patient age: report from the SENTRY Antimicrobial Surveillance Program (2008-2009). Diagn MicrobiolInfect Dis. 2010;68(3):278-83.

23. Melhem MS, Bertoletti A, Lucca HR, Silva RB, Meneghin FA, Szeszs MW. Use of the VITEK 2 system to identify and test the antifungal susceptibility of clinically relevant yeast species.Braz J Microbiol. 2014;44(4):1257-66

24. Ochiuzzi ME, Arechavala A, Guelfand L, Maldonado I, Soloaga R. Evaluation of the VITEK 2 system (AST-YSO1 cards) for antifungal susceptibility testing against different Candida species. Rev Argent Microbiol. 2014;46(2):111-8.

25. Organización Panamericana de la Salud/Organización Mundial de la Salud. Alerta epidemiológica sobre brotes de Candidaauris en servicios de atención a la salud, 2016. Disponible en: https://www. paho.org/hq/dmdocuments / 2016/2016-oct-3-phe-alerta-epicandida-auris.pdf Acceso el 20 de setiembre 2019.

Manuscrito recibido el 4 de octubre de 2019. Aceptado para su publicación, tras revisión, el 7 de febrero de 2020 . 


\section{Identification and susceptibility profile of Candida species isolated from hemocultures in hospitals in Paraguay}

SUMMARY

Keywords

Objective. To report the species identified in candidemia isolated in hospitals in Paraguay, their distribution by age groups, and their susceptibility to fluconazole, voriconazole, and amphotericin B.

Methods. Retrospective study of candidemia reported to the Central Health Public Laboratory by seven hospitals between 2010 and 2018. Identification and susceptibility testing were performed using the automated Vitek $2^{\circledR}$ system.

Results. 520 cases of candidiasis were reported. The prevalent species were Candida albicans (34.4\%), C. parapsilosis (30.4\%), C. tropical (25.4\%), C. glabrata (4.8\%), and C. krusei (2.1\%). Less frequent species were: C. lusitaniae (1.2\%), C. guilliermondii (1\%), C. famata $(0.3 \%)$, C. rugous $(0.2 \%)$, and C. kefyr $(0.2 \%)$. Candidiasis was most prevalent in the elderly (48.5\%) and adults (29.9\%). C. parapsilosis was most frequent in newborns and infants, and C. tropical in pediatric patients; $2.8 \%$ and $0.6 \%$ of $C$. albicans and $4.4 \%$ and $2.5 \%$ of $C$. parapsilosis were resistant, respectively, to fluconazole and voriconazole; $8 \%$ of $C$. glabrata were resistant to fluconazole and two isolations showed a minimum inhibitory concentration for voriconazole greater than $0.25 \mathrm{mg} / \mathrm{L}$.

Conclusions. This is the first report on candidemias in hospitals of Paraguay. Together with other studies, it will improve knowledge about this infection in the country.

Candidemia; antifungal agents; fluconazole; voriconazole; Paraguay

\section{Identificação e perfil de sensibilidade de Candida spp. isoladas de hemoculturas em hospitais no Paraguai}

RESUMO

Objetivo. Relatar as espécies identificadas em candidemias isoladas em hospitais do país, apresentar a sua distribuição por faixa etária e determinar a sua sensibilidade ao fluconazol, voriconazol e anfotericina $B$.

Métodos. Estudo retrospectivo de candidemias notificadas por sete hospitais ao Laboratório Central de Saúde Pública no período de 2010 a 2018. A identificação e o antifungigrama foram realizados pelo sistema automatizado Vitek ${ }^{\circledR}$.

Resultados. Foram notificados 520 casos de candidemia. As espécies predominantes foram Candida albicans $(34,4 \%)$, C. parapsilosis $(30,4 \%)$, C. tropicalis $(25,4 \%)$, C. glabrata $(4,8 \%)$ e C. krusei $(2,1 \%)$. Outras espécies menos frequentes foram: C. lusitaniae (1,2\%), C. guilliermondii (1\%), C. famata (0,3\%), C. rugosa $(0,2 \%)$ e $C$. kefyr $(0,2 \%)$. As candidemias em pacientes idosos $(48,5 \%)$ e adultos $(29,9 \%)$ foram as mais prevalentes. C. parapsilosis foi a espécie mais frequente em recém-nascidos e lactentes, e C. tropicalis predominou em pacientes pediátricos. Ao todo, 2,8\% e 0,6\% dos casos de C. albicans e 4,4\% e 2,5\% dos de C. parapsilosis foram resistentes a fluconazol e voriconazol, respectivamente. Além disso, $8 \%$ dos casos de C. glabrata foram resistentes ao fluconazol, e duas amostras isoladas apresentaram uma concentração inibitória mínima para voriconazol acima de 0,25 mg/L.

Conclusões. Este é o primeiro estudo sobre candidemias em hospitais do Paraguai, o que, juntamente com outros estudos, permitirá conhecer melhor esta infecção no país.

Palavras-chave Candidemia; antifúngicos; fluconazol; voriconazol; Paraguai. 